\title{
Experimental violation of Bell's inequality by local classical variables
}

\author{
Luigi Accardi and Massimo Regoli
}

\author{
Centro V. Volterra, Università di Roma Tor Vergata, Roma, Italy, \\ accardi@volterra.mat.uniroma2.it, WEB page: http://volterra.mat.uniroma2.it
}

\section{DESCRIPTION OF THE EXPERIMENT}

We describe a simple experiment in which two experimenters, by performing independent, local, binary choices on a common source of randomness and computing the empirical correlations among their results, arrive to a violation of Bell's inequalities.

Let $D$ denote the unit disk in the plane. On $D$ we define functions $S_{\alpha}^{(1)}, S_{\beta}^{(2)}$, with values \pm 1 , as follows: for any angle $\alpha \in[0,2 \pi)$, given the point $p$ in the disk, one rotates this point clockwise of an angle $\alpha$ and defines $S_{\alpha}^{(1)}(p)$ to be +1 if the rotated $p$ is in the upper half plane, --1 if it is in the lower half plane. $S_{\alpha}^{(2)}$ is defined by the singlet condition: $S_{\alpha}^{(1)}=-S_{\alpha}^{(2)}$.

First step: generate random points

an algorithm produces random points, $p_{1}, \ldots, p_{N}$, uniformly distributed in the unit circle in the plane.

\section{Second step: local choice of directions}

Each observer (Obs 1, Obs 2 in the program) chooses independently of the other one which observable to measure.

\section{Third step: local measurements}

The central unit sends the sequence $\left(p_{j}\right)$ of random points to the experimenters and the observer 1 (resp. 2) evaluates the function $S_{a}^{1}\left(p_{j}\right)$ (resp. $\left.S_{b}^{2}\left(p_{j}\right)\right), j=$ $1, \ldots, N$. The evaluation must be done on the same point, corresponding to the experimental fact that, in the EPR type experiments each measurement must be done on the same singlet pair. A measurement of the pair $\left(S_{\alpha}^{(1)}, S_{\gamma}^{(2)}\right)$ is statistically indistinguishable from a measurement of the pair $\left(S_{\pi+\alpha}^{(1)}, S_{\pi+\gamma}^{(2)}\right)$ so we always choose the pair $\left(S_{\alpha}^{(1)}, S_{\pi+\beta}^{(2)}\right)$ with $0 \leq \alpha, \beta<\pi$.

Fourth step: exchange of information after the measurement

$$
\begin{aligned}
& \text { CP519, Statistical Physics, edited by M. Tokuyama and H. E. Stanley } \\
& \text { (c) 2000 American Institute of Physics 1-56396-940-8/00/\$17.00 }
\end{aligned}
$$


The central unit is informed of the results and calculates the 3 correlations involved in the Bell inequality:

$$
\begin{aligned}
\left\langle S_{a}^{(1)} S_{b}^{(2)}\right\rangle & =\frac{1}{N} \sum_{j=1}^{N} S_{a}^{(1)}\left(p_{j}^{(1)}\right) S_{b}^{(2)}\left(p_{j}^{(1)}\right) \\
\left\langle S_{b}^{(2)} S_{c}^{(1)}\right\rangle & =\frac{1}{N} \sum_{j=1}^{N} S_{c}^{(1)}\left(p_{j}^{(2)}\right) S_{b}^{(2)}\left(p_{j}^{(2)}\right) \\
\left\langle S_{a}^{(1)} S_{c}^{(2)}\right\rangle & =\frac{1}{N} \sum_{j=1}^{N} S_{a}^{(1)}\left(p_{j}^{(3)}\right) S_{c}^{(2)}\left(p_{j}^{(3)}\right)
\end{aligned}
$$

Notice that (both in the real and in the simulation experiment) the evaluation of the correlation (1) is done using the sequence $\left(p_{j}^{(1)}\right)$ of results of experiment (1), similarly correlation (2) uses the sequence $\left(p_{j}^{(2)}\right)$, and correlation (3) uses $\left(p_{j}^{(3)}\right)$. It is not relevant in which order one computes these correlations, e.g. one can choose at random every time which of the three pairs $(a, b),(b, c),(c, a)$ to measure (Aspect experiment): since the experiments are incompatible, the 3 sequences are uniquely determined.

Fifth step: computation of the empirical correlations

The central unit computes the 3 empirical correlations (1), (2), (3).

Sixth step: cleaning

In order to apply Bell's argument to the empirical data, it is necessary (cf. section (1) of [Ac99]) to clean these data as follows: all the points $p_{j}^{(1)}, p_{j}^{(2)}$ such that

$$
S_{b}^{(2)}\left(p_{j}^{(1)}\right) \neq S_{b}^{(2)}\left(p_{j}^{(2)}\right)
$$

have to be eliminated from the calculation of the empirical correlations (1), (2), (3).

An elementary argument of classical probability shows that, if the experiments are mutually independent this elimination does not alter significantly the empirical correlations. It is however surprising that, in the vast literature on the Bell inequality, the necessity of this "cleaning operation" has not been sufficiently emphasized .

Seventh step: computation of the cleaned correlations

After the "cleaning" one obtains two new sequences of points:

$$
p_{j_{1}}^{(1)}, \ldots, p_{j_{N_{1}}}^{(1)}, p_{j_{1}}^{(2)}, \ldots, p_{j_{N_{1}}}^{(2)}
$$

with the property that

$$
S_{b}^{(2)}\left(p_{j_{\alpha}}^{(1)}\right)=S_{b}^{(2)}\left(p_{j_{\alpha}}^{(2)}\right) ; \quad \alpha=1, \ldots, N_{1}
$$


The central unit computes the new correlations with the "cleaned" sequences and compares them with the original ones (1), (2). We have checked that the difference between the original correlations and the "cleaned" ones is of orders of magnitude smaller than the violation of the inequality. Therefore this violation cannot be attributed to the "cleaning" operation which, on the other hand is not an assumption related to our experiment, but only represents the explicitation of an assumption hidden in any comparison of the Bell inequality with the experimental data. For more informations on this point, cf. [AcRe99a].

Eigth step: checking the Bell inequality

According to the Bell inequality one should have

$$
\left|\left\langle S_{a}^{(1)} S_{b}^{(2)}\right\rangle-\left\langle S_{c}^{(1)} S_{b}^{(2)}\right\rangle\right| \leq 1+\left\langle S_{a}^{(1)} S_{c}^{(2)}\right\rangle
$$

The program compares the difference

$$
\left|\left\langle S_{a}^{(1)} S_{b}^{(2)}\right\rangle-\left\langle S_{c}^{(1)} S_{b}^{(2)}\right\rangle\right|-1-\left\langle S_{a}^{(1)} S_{c}^{(2)}\right\rangle
$$

with zero. Violation of Bell's inequality corresponds to a value of the difference (8) strictly greater than zero. The simulation experiments show that, if the directions $a, b, c$ are chosen so that $a$ coincides with the $x$-axis and the directions $b, c$ intersect the second and fourth quadrant in such a way that $\widehat{c a}>\widehat{a c}$ then the difference (8) is strictly positive, hence the Bell inequality is violated.

The reason of the violation is the following: let us denote $\widehat{a b}$ the sector of the $(-,-)$ concordance in the correlation $\left\langle S_{a}^{(1)} S_{b}^{(2)}\right\rangle$. Similarly we denote $\widehat{c b}$ the sector of the $(-,-)$ concordance in the correlation $\left\langle S_{c}^{(1)} S_{b}^{(2)}\right\rangle$ and $\widehat{a c}$ the sector of the $(-,-)$ concordance in the correlation $\left\langle S_{a}^{(1)} S_{c}^{(2)}\right\rangle$. With these notations the correlations (5), $(6),(7)$ are given by:

$$
\begin{aligned}
& \left\langle S_{a}^{(1)} S_{b}^{(2)}\right\rangle=-1+4 \widehat{a b} \\
& \left\langle S_{c}^{(1)} S_{b}^{(2)}\right\rangle=-1+4 \widehat{c b} \\
& \left\langle S_{a}^{(1)} S_{c}^{(2)}\right\rangle=-1+4 \widehat{a c}
\end{aligned}
$$

So the two sides of the Bell inequality are respectively:

$$
\begin{gathered}
\left|\left\langle S_{a}^{(1)} S_{b}^{(2)}\right\rangle-\left\langle S_{c}^{(1)} S_{b}^{(2)}\right\rangle\right|=4|\widehat{a b}-\widehat{c b}|=4(\widehat{c b}-\widehat{a b}) \\
1+\left\langle S_{a}^{(1)} S_{c}^{(2)}\right\rangle=4 \widehat{a c}
\end{gathered}
$$

and we are reduced to compare $\widehat{c b}-\widehat{a b}=\widehat{c a} \quad$ and $\quad \widehat{a c}$. Therefore if one chooses the axes so that $\widehat{c a}>\widehat{a c}$, it will follow that

$$
\left|\left\langle S_{a}^{(1)} S_{b}^{(2)}\right\rangle-\left\langle S_{c}^{(1)} S_{b}^{(2)}\right\rangle\right|>1+\left\langle S_{a}^{(1)} S_{c}^{(2)}\right\rangle
$$

which violates the Bell inequality (14). 


\section{REFERENCES}

1. [Ac95] L. Accardi: Can mathematics help solving the interpretational problems of quantum theory? Il Nuovo Cimento 110 B (1995) 685-721, cf. also: Mesoscopic Physics and fundamental problems in quantum mechanics, C.Di Castro, F.Guerra, G. Jona-Lasinio (1995)

2. [Ac97] Accardi L.: Urne e camaleonti. Dialogo sulla realtà, le leggi del caso e la teoria quantistica. Il Saggiatore (1997). Japanese translation by Kayoko Awaya, Maruzen (2000)

3. [Ac99] Accardi L.: On the EPR paradox and the Bell inequality Volterra Preprint (1998) N. 350.

4. [AcRe99a] Luigi Accardi, Massimo Regoli: Quantum probability and the interpretation of quantum mechanics: a crucial experiment, Invited talk at the workshop: "The applications of mathematics to the sciences of nature: critical moments and aspetcs", Arcidosso June 28-July 1. To appear in the proceedings of the workshop, Preprint Volterra N. 399 
Copyright $\odot 2003$ EBSCO Publishing 
Copyright of AIP Conference Proceedings is the property of American Institute of Physics and its content may not be copied or emailed to multiple sites or posted to a listserv without the copyright holder's express written permission. However, users may print, download, or email articles for individual use. 
Copyright of AIP Conference Proceedings is the property of American Institute of Physics and its content may not be copied or emailed to multiple sites or posted to a listserv without the copyright holder's express written permission. However, users may print, download, or email articles for individual use. 